\title{
Observation of Polarized Positrons from an Undulator-Based Source
}

G. Alexander, ${ }^{1}$ J. Barley, ${ }^{2}$ Y. Batygin, ${ }^{3}$ S. Berridge, ${ }^{4}$ V. Bharadwaj, ${ }^{3}$ G. Bower, ${ }^{3}$ W. Bugg, ${ }^{4}$ F.-J. Decker, ${ }^{3}$ R. Dollan, ${ }^{5}$ Y. Efremenko, ${ }^{4}$ V. Gharibyan, ${ }^{6,7}$ C. Hast, ${ }^{3}$ R. Iverson, ${ }^{3}$ H. Kolanoski, ${ }^{5}$ J. Kovermann, ${ }^{8}$ K. Laihem, ${ }^{9}$ T. Lohse, ${ }^{5}$

K. T. McDonald, ${ }^{10}$ A. A. Mikhailichenko, ${ }^{2}$ G. A. Moortgat-Pick, ${ }^{11}$ P. Pahl, ${ }^{6}$ R. Pitthan,${ }^{3}$ R. Pöschl,${ }^{6}$

E. Reinherz-Aronis, ${ }^{1}$ S. Riemann, ${ }^{9}$ A. Schälicke, ${ }^{9}$ K. P. Schüler,${ }^{6}$ T. Schweizer,${ }^{5}$ D. Scott, ${ }^{12}$

J. C. Sheppard, ${ }^{3}$ A. Stahl, ${ }^{8}$ Z. M. Szalata, ${ }^{3}$ D. Walz, ${ }^{3}$ and A. W. Weidemann ${ }^{3}$

${ }^{1}$ Tel-Aviv University, Tel Aviv 69978, Israel

${ }^{2}$ Cornell University, Ithaca, New York 14853, USA

${ }^{3}$ SLAC, Menlo Park, California 94025, USA

${ }^{4}$ University of Tennessee, Knoxville, Tennessee 37996, USA

${ }^{5}$ Institut für Physik, Humboldt-Universität zu Berlin, D-12489 Berlin, Germany

${ }^{6}$ DESY, D-22607 Hamburg, Germany

${ }^{7}$ YerPhI, Yerevan 375036, Armenia

${ }^{8}$ RWTH Aachen, D-52056 Aachen, Germany

${ }^{9}$ DESY, D-15738 Zeuthen, Germany

${ }^{10}$ Joseph Henry Laboratories, Princeton University, Princeton, New Jersey 08544, USA

${ }^{11}$ University of Durham, Durham, DH1 3LE, United Kingdom

${ }^{12}$ STFC Daresbury Laboratory, Daresbury, Warrington, Cheshire, WA4 4AD, United Kingdom

(Received 8 March 2008; published 29 May 2008)

An experiment (E166) at the Stanford Linear Accelerator Center has demonstrated a scheme in which a multi-GeV electron beam passed through a helical undulator to generate multi-MeV, circularly polarized photons which were then converted in a thin target to produce positrons (and electrons) with longitudinal polarization above $80 \%$ at $6 \mathrm{MeV}$. The results are in agreement with GEANT4 simulations that include the dominant polarization-dependent interactions of electrons, positrons, and photons in matter.

DOI: 10.1103/PhysRevLett.100.210801

A polarized positron beam would enhance the physics capability of a TeV-scale $e^{+} e^{-}$linear collider [1]. Polarized positrons can be produced via the pairproduction process initiated by circularly polarized photons [2]. In a scheme proposed by Balakin and Mikhailichenko [3] a multi-GeV electron beam is passed through a helical undulator [4] to generate the needed multi-MeV photons with circular polarization. Alternatively, the circularly polarized photons can be produced by laser backscattering off an electron beam [5,6]. An experiment (E166) has been performed to demonstrate that the undulator-based scheme can produce polarized positron beams of sufficient quality for use at the proposed International Linear Collider (ILC) [7]. The main elements of the experiment were the Stanford Linear Accelerator Center (SLAC) linac [8], the Final Focus Test Beam (FFTB) [9], a pulsed helical undulator, and detectors to measure the photon and positron polarizations [10], as shown schematically in Fig. 1.

The experiment operated with an electron beam energy of $46.6 \pm 0.1 \mathrm{GeV}$ at a repetition rate of $10 \mathrm{~Hz}$ with $1-4 \times$ $10^{9} \mathrm{e} / \mathrm{pulse}$. The normalized beam emittances were $\gamma \epsilon_{x}\left(\gamma \epsilon_{y}\right) \approx 2.2(0.5) \times 10^{-5} \mathrm{~m} \mathrm{rad}$, and the transverse spot size was tuned to $\sigma_{x} \approx \sigma_{y} \approx 35 \mu \mathrm{m}$ at the $1-\mathrm{m}$ long undulator whose aperture was only $0.9 \mathrm{~mm}$. After passing through the undulator, the primary electron beam was deflected away from the photon beam by a string of permanent magnets (D1). A circularly polarized photon
PACS numbers: 07.77.Ka, 13.88.+e, 29.27.Hj, 41.75.Fr

beam of peak energy $\approx 8 \mathrm{MeV}$ was created in the undulator and then drifted approximately $35 \mathrm{~m}$ to the diagnostic detectors, shown in the lower part of Fig. 1 and in greater detail in Fig. 2.
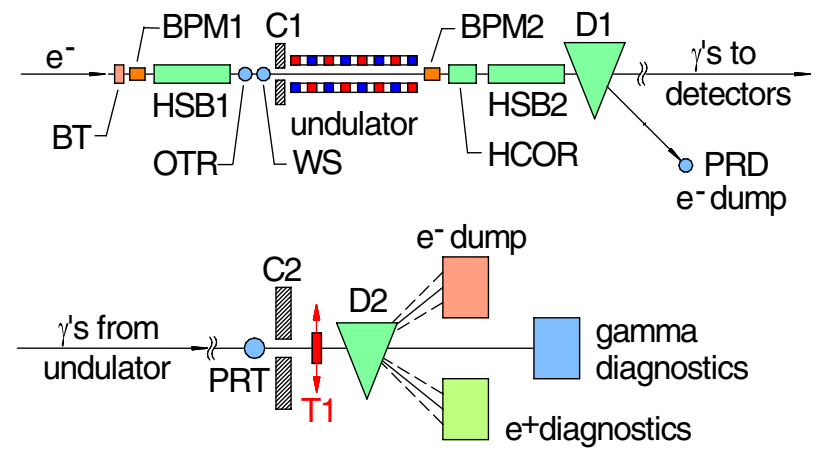

FIG. 1 (color online). Conceptual layout (not to scale) of the E166 experiment. A 46.6-GeV electron beam entered from the left and was deflected by magnet D1 after traversing the undulator. Part of the beam of $\approx 8-\mathrm{MeV}$ circularly polarized photons created in the undulator was converted to positrons in a target $35 \mathrm{~m}$ downstream of the undulator, and the rate and polarization of the positrons and unconverted photons were subsequently diagnosed in the spectrometer D2. BPM = beam-position monitor, $\mathrm{BT}=$ beam toroid, $\quad \mathrm{C}=$ collimator, $\quad \mathrm{HCOR}=$ horizontal-correction magnet, HSB = hard-soft-bend magnet, OTR $=$ optical-transition-radiation monitor, $\quad \mathrm{PR}=$ beam-profile monitor, $\mathrm{T} 1=$ target, $\mathrm{WS}=$ wire scanner. 


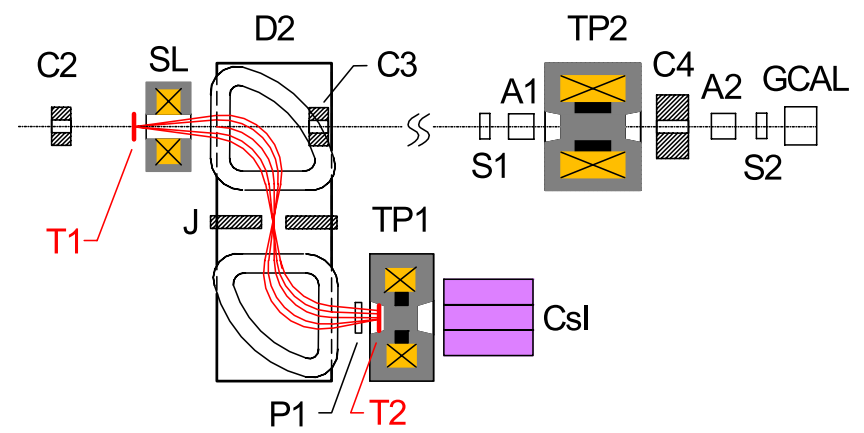

FIG. 2 (color online). Schematic of the photon and positron diagnostics. A1, A2 = aerogel Čerenkov detectors, C2 - C4 = collimators, $\mathrm{D} 2=$ dipole spectrometer magnet, $\quad \mathrm{Csl}=3 \times 3$ array of CsI crystals, $\mathrm{GCAL}=\mathrm{Si}-\mathrm{W}$ calorimeter, $\mathrm{J}=$ movable $\mathrm{W}$ jaws, $\mathrm{P} 1, \mathrm{~S} 1, \mathrm{~S} 2=$ Si-diode detectors, $\mathrm{SL}=$ solenoid lens, $\mathrm{T} 1=$ positron production target, $\quad \mathrm{T} 2=$ reconversion target, TP1 = positron transmission polarimeter solenoid, $\quad$ TP2 = photon transmission polarimeter solenoid. The detectors were encased in lead and tungsten shielding (not shown).

The photon beam impinged upon a 0.2-radiation-length tungsten target $\mathrm{T} 1$ to produce positrons and electrons which were separated in spectrometer D2, and the polarization and rate of the positrons were measured in transmission polarimeter TP1 [11]. The unconverted photons were monitored in a second transmission polarimeter, TP2.

The undulator had bifilar, helical windings of wires of cross section $0.6 \times 0.6 \mathrm{~mm}^{2}$ with currents $(2.3 \mathrm{kA}$ in a $12 \mu$ s pulse) flowing in opposite directions, resulting in a transverse magnetic field whose direction rotated with period $2.54 \mathrm{~mm}$ and whose strength was $0.71 \mathrm{~T}$ on axis, corresponding to an undulator strength parameter of $K=$ 0.17 . The calculated energy spectrum and longitudinal polarization of the photons produced by the undulator are shown in Fig. 3. For an electron beam energy of $46.6 \mathrm{GeV}$ and $K=0.17$ the first-harmonic photon energy cutoff is $E_{\gamma}=7.9 \mathrm{MeV}$, at which energy the longitudinal polarization $P_{\gamma}$ is 0.98 , differing from unity due to the small admixture of second harmonic photons.

The photon beam was monitored in a transmission polarimeter TP2, indicated in the right side of Fig. 2. The flux of photons was determined by aerogel Čerenkov counters, A2, A2, and by silicon-diode detectors, S1, S2, before and after a 15-cm-long cylinder of iron whose axial magnetization was reversed periodically. The total energy of photons that passed through the iron cylinder was monitored in a W-plate calorimeter GCAL read out by interleaved Si diodes. The photon flux at full undulator current, observed in detector $\mathrm{S} 1$ with the pair-production target T1 removed, was $0.071 \pm 0.007$ photons/beam electron, which value is only $20 \%$ of expectations, likely due to misalignment of collimator $\mathrm{C} 2$. When the undulator current was reduced, the photon flux showed the expected quadratic dependence on the current.

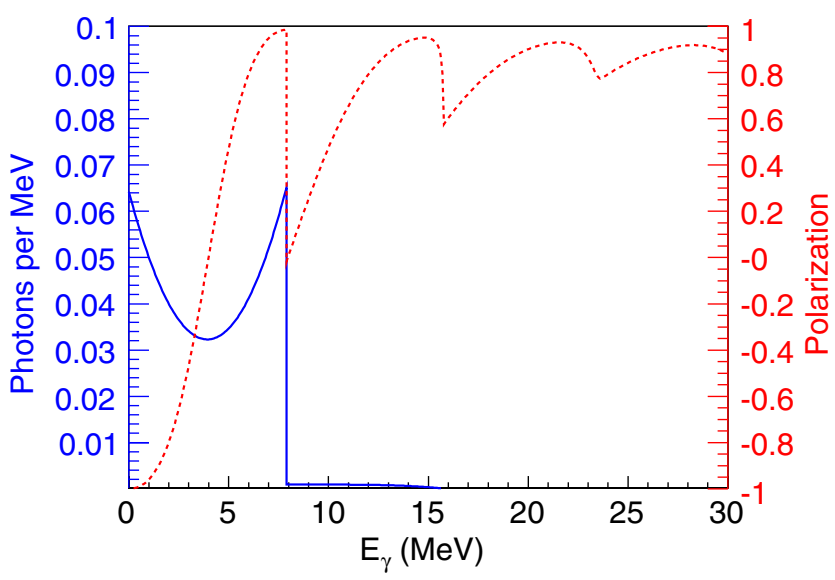

FIG. 3 (color online). Solid line: calculated photon number spectrum per beam electron of undulator radiation integrated over angle, plotted as a function of photon energy $E_{\gamma}$ for electron beam energy $46.6 \mathrm{GeV}$, undulator period $2.54 \mathrm{~mm}$ and undulator strength parameter $K=0.17$. The peak energy of the firstharmonic (dipole) radiation was 7.9 MeV. Dashed line: longitudinal polarization $P_{\gamma}$ of the undulator radiation as a function of energy.

The asymmetry $\delta_{\gamma}=\left(S_{\gamma}^{-}-S_{\gamma}^{+}\right) /\left(S_{\gamma}^{-}+S_{\gamma}^{+}\right)$in the observed signals $S_{\gamma}^{ \pm}$of photons transmitted through the iron cylinder was $0.0331 \pm 0.0012$ (stat) \pm 0.0063 (syst) using aerogel detector A2, $0.0367 \pm 0.0007$ (stat) \pm 0.0040 (syst) using calorimeter GCAL, and $0.0388 \pm 0.0006$ (stat) \pm 0.0016 (syst) using Si-diode detector S2. A simulation that combined the energy and polarization distributions shown in Fig. 3 with the spin dependence of Compton scattering of the polarized photons off polarized atomic electrons in the magnetized iron [12] calculated asymmetries of 0.036 for detector A2 (assuming a Čerenkov threshold of $3.8 \mathrm{MeV}$ ), 0.035 for GCAL, and 0.034 for S2.

Positrons (and electrons) produced from undulator photons in the $\mathrm{W}$ target $\mathrm{T} 1$ were focused to a parallel beam by solenoid lens SL and then energy selected and separated from the electrons and unconverted photons in spectrometer D2 consisting of a pair of dipole magnets, shown in Fig. 2. The energy spread of positrons at the reconversion target T2 was 5\% (FWHM). The positron flux (typically $2-6 \times 10^{4} /$ pulse with undulator on and $1 \%$ of this with undulator off) was monitored at this location by Si-diode detector P1. The polarization of the positrons was determined by first reconverting them into polarized photons by a 0.5-radiation-length $\mathrm{W}$ disk, and then using transmission polarimeter TP1 to measure the longitudinal polarization of the photons. This polarimeter consisted of a 7.5-cm-long magnetized iron cylinder followed by a $3 \times 3$ array of CsI crystals.

Data were collected with the undulator on and off during successive electron beam pulses. The sign of the magnetization of polarimeter TP1 (and that of TP2 as well) was reversed after every 1500 undulator-on beam pulses. 
Beam-off and target-out runs were interspersed throughout the data sets. Data were taken with positrons at five energies from 4.6 to $7.4 \mathrm{MeV}$, and with electrons at a single energy $(6.7 \mathrm{MeV})$ for which the current in dipole spectrometer D2 (but not that in solenoid lens SL) was reversed. Data samples for each energy ranged from $2-20 \times 10^{5}$ beam pulses and a total of more than $8 \times$ $10^{6}$ events were recorded during the experiment.

The distribution of photon energies from reconverted positrons as observed in the central CsI crystal for central positron energy of $6.7 \mathrm{MeV}$ is shown in Fig. 4 for undulator-on and undulator-off beam pulses. Approximately 30 photons from reconverted positrons were observed each pulse above a background of a similar number of $\mathrm{MeV}$ particles from showers of beam electrons that scraped the undulator tube.

The positron (or electron) polarization is derived from the asymmetry

$$
\delta_{e^{ \pm}}=\left(S_{\mathrm{CSI}}^{-}-S_{\mathrm{CSI}}^{+}\right) /\left(S_{\mathrm{CsI}}^{-}+S_{\mathrm{CSI}}^{+}\right)
$$

of signals $S_{\mathrm{CSI}}^{ \pm}$that are proportional to the (integrated) energies $E_{\mathrm{C} S I}^{ \pm}$of reconverted photons observed in the central CsI crystal for the two signs of axial magnetization of polarimeter TP1. The outer eight crystals of the CsI array were not used in the final analysis because of poorer signalto-background ratio. The energy calibration of the crystals was maintained by data collected with radioactive sources embedded in the array. The photon energies $E_{\mathrm{CSI}}^{ \pm}$were corrected for background using the undulator-off data, and normalized to the rates observed in the Si-diode detector $\mathrm{P} 1$ according to

$$
S_{\mathrm{CSI}}=\frac{1}{N_{\mathrm{on}} N_{\mathrm{off}}} \sum_{i=1}^{N_{\mathrm{on}}} \sum_{j=1}^{N_{\mathrm{off}}} \frac{E_{\mathrm{Cs}, i}^{\mathrm{on}}-E_{\mathrm{Cs}, j}^{\mathrm{off}} \frac{I_{i}^{\text {on }}}{I_{j}^{\text {off }}}}{\mathrm{P} 1_{i}^{\text {on }}-\mathrm{P} 1_{j}^{\text {off }} \frac{I_{i}^{\text {on }}}{I_{j}^{\text {off }}}},
$$

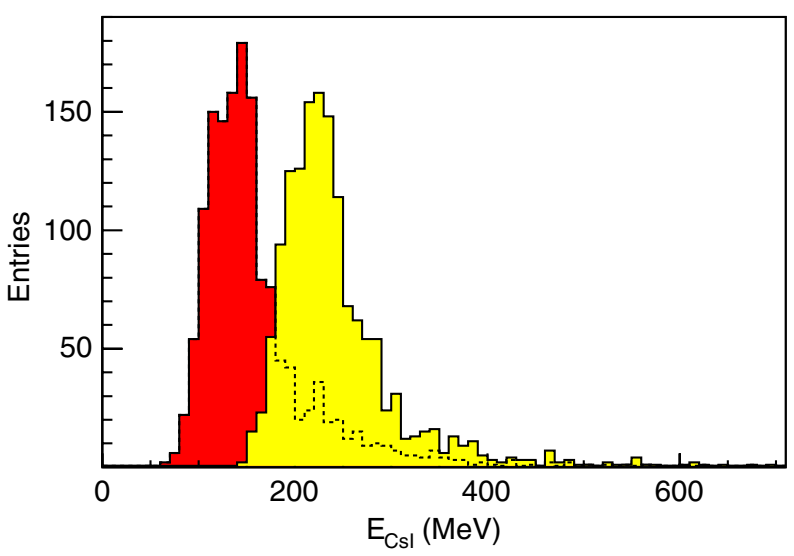

FIG. 4 (color online). Distributions of energy $E_{\mathrm{CsI}}$ observed in the central CsI crystal of the positron polarimeter from individual electron beam pulses with undulator-on (right peak) and undulator-off (left peak). The central positron energy was $6.7 \mathrm{MeV}$. where $N_{\text {on }} \approx N_{\text {off }} \approx 1500$ are the numbers of events with undulator-on and off in data sets with \pm magnetization of polarimeter TP1, $I$ is the number of $46.6-\mathrm{GeV}$ electrons as measured in beam toroid BT, and P1 is the signal observed in that Si-diode detector. Terms in Eq. (2) more than 2 standard deviations from the mean were discarded to stabilize the averaging procedure against the effect of outliers caused by occasional off-energy electron beam pulses.

The asymmetry (1) was calculated for each pair of 1500 undulator-on beam pulses with opposite magnetization of the polarimeter, as shown in Fig. 5 for data collected with a central positron energy of $6.1 \mathrm{MeV}$. Asymmetries more than 3 standard deviations from the average were discarded. The averaged asymmetries were typically $1 \%$, as listed in Table I. Without the normalization (2) to the rates in detector $\mathrm{P} 1$, the asymmetries would have been about $10 \%$ smaller for positrons and $40 \%$ larger for electrons, which indicates differing effects on these particle types of their interactions with the stray fields of the solenoid lens $\mathrm{SL}$, the spectrometer magnet D2, and the polarimeter magnet TP1. Alternative normalization procedures yielded results consistent with those given in Table I. Use of all nine CsI crystals in the analysis yielded similar results but with larger uncertainties due to the relatively larger backgrounds in the outer crystals.

The longitudinal polarization $P_{e^{ \pm}}$of the positrons (electrons) is deduced from the measured asymmetry $\delta_{e^{ \pm}}$using the relation

$$
P_{e^{ \pm}}=\frac{\delta_{e^{ \pm}}}{A_{e^{ \pm}} P_{e^{-}}^{\mathrm{Fe}}},
$$

where $P_{e^{-}}^{\mathrm{Fe}}=0.0695 \pm 0.0021$ is the longitudinal polarization of the atomic electrons in the iron cylinder, and $A_{e^{ \pm}}$ is the analyzing power determined by numerical simulation. The latter was performed with an enhanced version of the GEANT4 toolkit [13] that included six new routines to deal with circularly polarized photon beams and longitudinally polarized electron beams [14]: for Compton scattering, Møller or Bhabha scattering, and electron-positron annihilation the dependence of the cross section on beam

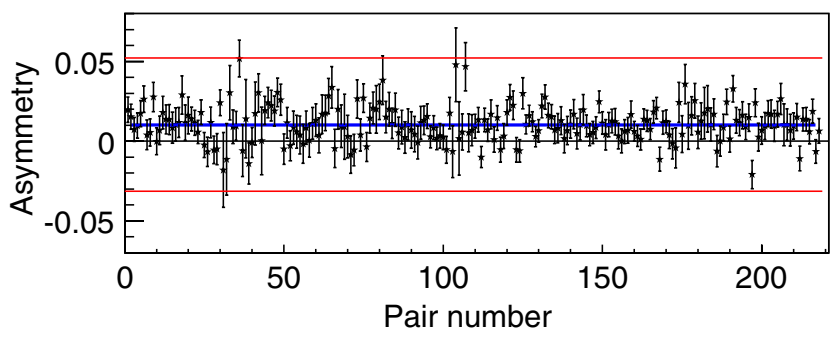

FIG. 5 (color online). Positron-induced asymmetries $\delta_{e^{+}}$in the central CsI crystal of the positron polarimeter for 220 pairs of 1500 undulator-on beam pulses with opposite magnetization in the polarimeter. The central positron energy was $6.1 \mathrm{MeV}$. The average asymmetry was 0.0108 , as indicated by the horizontal line. 
TABLE I. The asymmetries $\delta_{e^{ \pm}}($in $\%)$ observed in the transmission polarimeter TP1, and the corresponding analyzing powers $A_{e^{ \pm}}$and longitudinal polarizations $P_{e^{ \pm}}$(in $\%$ ) as a function of energy $E_{e^{ \pm}}$in $\mathrm{MeV}$.

\begin{tabular}{cccc}
\hline \hline$E_{e^{ \pm}}$ & $\delta \pm \sigma_{\delta}($ stat $)$ & $A$ & $P \pm \sigma_{P}($ stat $) \pm \sigma_{P}($ syst $)$ \\
\hline $4.6\left(e^{+}\right)$ & $0.69 \pm 0.17$ & 0.150 & $66 \pm 16 \pm 8$ \\
$5.4\left(e^{+}\right)$ & $0.96 \pm 0.08$ & 0.156 & $89 \pm 8 \pm 9$ \\
$6.1\left(e^{+}\right)$ & $1.08 \pm 0.06$ & 0.162 & $96 \pm 6 \pm 10$ \\
$6.7\left(e^{+}\right)$ & $0.92 \pm 0.08$ & 0.165 & $80 \pm 7 \pm 9$ \\
$6.7\left(e^{-}\right)$ & $0.94 \pm 0.05$ & 0.153 & $88 \pm 5 \pm 15$ \\
$7.4\left(e^{+}\right)$ & $0.89 \pm 0.20$ & 0.169 & $76 \pm 17 \pm 12$ \\
\hline \hline
\end{tabular}

and target polarization was modeled; in addition, the polarization transfer from initial- to final-state particles in bremsstrahlung, electron-positron pair annihilation and creation, and the photoelectric effect was evaluated. The relative systematic uncertainty on the analyzing power is estimated to be $7 \%$.

The asymmetries $\delta$, the analyzing powers $A$, and the longitudinal polarizations $P_{e^{ \pm}}$of electrons and positrons deduced using Eq. (3) are listed in Table I, and the polarizations are shown together with simulations in Fig. 6 as a function of particle energy. The shift between the curves arises because for photon energies that peak near $E_{\gamma}=$ $7.9 \mathrm{MeV}$ the maximum energy of a positron from pair production is $E_{e^{+}}=E_{\gamma}-m c^{2} \approx 7.4 \mathrm{MeV}$, while electrons from Compton scattering and the photoelectric effect have maximum energies $E_{e^{-}}^{\mathrm{C}} \approx E_{\gamma}+m c^{2} / 2 \approx$ $8.2 \mathrm{MeV}$ and $E_{e}^{\mathrm{PE}}=E_{\gamma}+m c^{2} \approx 8.4 \mathrm{MeV}$, respectively, where $m c^{2}=511 \mathrm{keV}$ is the rest energy of the electron.

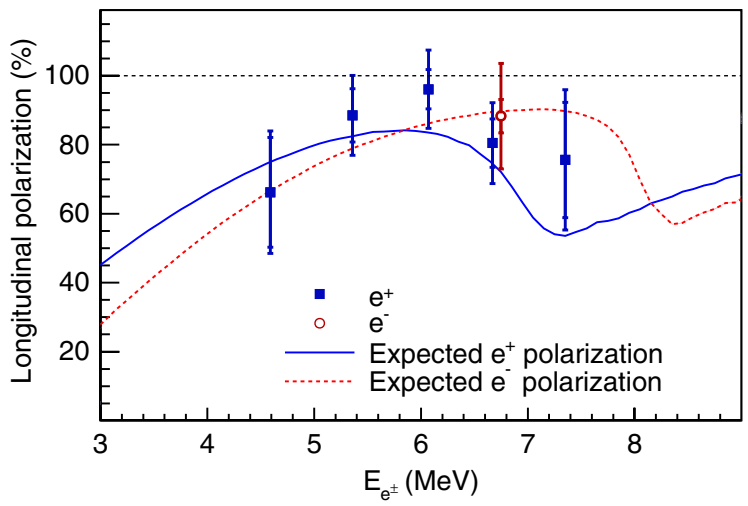

FIG. 6 (color online). Longitudinal polarization $P_{e^{ \pm}}$as a function of energy $E_{e^{ \pm}}$of positrons and electrons as determined from the asymmetries $\delta_{e^{ \pm}}$observed in the central CsI crystal. The smaller error bars show the statistical uncertainty, and the larger bars indicate the statistical and systematic uncertainties combined in quadrature. Also shown are predictions by a GEANT4 simulation of the experiment.
The uncertainties shown in the figure include both statistical and systematic effects where the latter were estimated from studies of the effects of non-Gaussian fluctuations and outlier rejection, from the quality cuts on the beam current, of the pairing of sets of 1500 beam pulses, of background correction, and of the stray-field-induced asymmetry at the counter P1 used for signal normalization.

The results of this experiment are in agreement with GEANT4 simulations that positron polarization of $80 \%$ is obtainable at $\mathrm{MeV}$ energies when $\mathrm{GeV}$ electrons pass through a helical undulator, producing $\mathrm{MeV}$ photons that are converted in a thin target. The polarization extensions to GEANT4 provide a basis for optimization of the ILC positron source, and for other applications of polarization transfer, such as polarimetry. The technique of undulatorbased production of polarized positrons, demonstrated in this experiment, can be scaled up to provide polarized positron (and electron) beams for the next generation of linear colliders [7].

This work was supported in part by DOE Contract No. DE-AC03-76SF00515, DOE Grants and Nos. DEFG05-91ER40627, DE-FG02-91ER40671, DE-FG0203ER41283, and DE-FG02-04ER41353, by NSF Grant No. PHY-0202078 (U.S.), by European Commission Contract No. RIDS-011899 (Germany), by the STFC (U.K.), and by ISF Contract No. 342/05 (Israel).

[1] G. Moortgat-Pick et al., Phys. Rep. 460, 131 (2008).

[2] H. Olsen and L. C. Maximon, Phys. Rev. 114, 887 (1959).

[3] V.E. Balakin and A. A. Mikhailichenko, Budker Institute of Nuclear Physics Report No. BINP 79-85, 1979.

[4] R. C. Wingerson, Phys. Rev. Lett. 6, 446 (1961).

[5] E. G. Bessonov and A.A. Mikhailichenko, Budker Institute of Nuclear Physics BINP 92-43, 1992.

[6] T. Omori et al., Phys. Rev. Lett. 96, 114801 (2006).

[7] International Linear Collider Reference Design Report, 2007, http://www.linearcollider.org/rdr

[8] R. Erickson et al., Report No. SLAC-R-714, 1984.

[9] M. Berndt et al., Report No. SLAC-376, 1991.

[10] G. Alexander et al., Nucl. Instrum. Methods Phys. Res., Sect. A (to be published).

[11] H. Schopper, Nucl. Instrum. 3, 158 (1958).

[12] H. Frauenfelder and A. Rossi, in Methods of Experimental Physics: Nuclear Physics, edited by L.C. L. Yuan and C.-S. Wu (Academic Press, New York, 1963), Part B5, p. 214.

[13] S. Agostinelli et al. (GEANT4 Collaboration), Nucl. Instrum. Methods Phys. Res., Sect. A 506, 250 (2003); J. Allison et al., IEEE Trans. Nucl. Sci. 53, 270 (2006).

[14] R. Dollan, K. Laihem, and A. Schälicke, Nucl. Instrum. Methods Phys. Res., Sect. A 559, 185 (2006); A. Schälicke, K. Laihem, and P. Starovoitov, Report No. DESY 07-202, 2007. 